\title{
Compatibility of Porous Chitosan Scaffold with the Attachment and Proliferation of human Adipose-Derived Stem Cells In Vitro
}

\author{
Gomathysankar $\mathrm{S}^{1}$, Halim AS ${ }^{1}$, Yaacob NS ${ }^{2}$, Noor $\mathrm{NM}^{1}$, Mohamed $\mathrm{M}^{1}$
}

Adipose-derived stem cells (ASCs) have potential applications in the repair and regeneration of various tissues and organs. The use of various scaffold materials as an excellent template for mimicking the extracellular matrix to induce the attachment and proliferation of different cell types has always been of interest in the field of tissue engineering because ideal biomaterials are in great demand. Chitosan, a marine polysaccharide, have wide clinical applications and it acts as a promising scaffold for cell migration and proliferation. ASCs, with their multi-differentiation potential, and chitosan, with its great biocompatibility with ASCs, were investigated in the present study.

ASCs were isolated and were characterized by two different methods: immunocytochemistry and flow cytometry, using the mesenchymal stem cell markers CD90, CD105, CD73 and CD29. The ASCs were then induced to differentiate into adipogenic, osteogenic and chondrogenic lineages. These ASCs were incorporated into a porous chitosan scaffold (PCS), and their structural morphology was studied using a scanning electron microscope and hematoxylin and eosin staining. The proliferation rate of the ASCs on the PCS was assessed using a PrestoBlue viability assay.

The results indicated that the PCS provides an excellent template for the adhesion and proliferation of ASCs. Thus, this study revealed that PCS is a promising biomaterial for inducing the proliferation of ASCs, which could lead to successful tissue reconstruction in the field of tissue engineering.

Key Words: Adipose-derived stem cells; Porous chitosan scaffold; Multi-differentiation; Proliferation; Structural morphology

\section{Introduction}

Stem cells have self-renewing capacity and are able to differentiate into single or multiple types of specialized stem cells. MSCs are the stem cells which are most widely used for various therapeutic purposes due to their ease of isolation from the organs and tissues where they reside ${ }^{[1]}$. Bone marrow, umbilical cord and adipose tissue are the main sources of MSCs. The stem cells that are derived from human adipose tissue are known as adipose-derived stem cells (ASCs), which have a similar phenotype and morphology as bone marrowderived $\mathrm{MSCs}^{[2]}$. ASCs are mostly obtained from liposuction procedures and also from tissue resected from the subcutaneous and visceral fat depots of white adipose tissue (WAT). ASCs from different origins have distinct inherent properties ${ }^{[3,4]}$. The acquisition of tissue is less complex and the cells are more abundant for ASCs than for bone marrow-derived MSCs. Because of their high proliferative and differentiative capacities, ASCs are capable of repairing vital tissues and organs, thereby replacing lost soft tissues. All of these remarkable properties make ASCs as the most promising cell type for tissue engineering and regenerative medicine ${ }^{[1,5,6]}$.

ASCs from subcutaneous adipose tissue were used in the present study. The selection of an artificial skin substitute is equally as important as the selection of an appropriate cell line because the success rate of the repair process depends on the interaction between the scaffold used and the selected cell line that is seeded on the scaffold. Recently, researchers have shown intensified interest in seeding stem cells on tissue-engineered scaffolds $^{[7-9]}$. Due to the various limitations of skin grafts and artificial implants, the purpose of developing biological substitutes or $3 \mathrm{D}$ scaffolds incorporating appropriate cell lines is better restoration of tissue function ${ }^{[10,11]}$. Because ASCs are easy to isolate and are abundantly available, they are the stem cells most commonly seeded on various biomaterials to study the efficacy of stem cells in repairing damaged tissues ${ }^{[12]}$.

\begin{abstract}
There is a wide range of natural and synthetic polymers available for use as scaffold materials. Selecting the appropriate biomaterial among these various types of biopolymers is crucial ${ }^{[13]}$. The combination of ASCs with appropriate biomaterials or skin substitutes is promising in the regeneration of damaged tissues. Several of the naturally derived biomaterials used for fat tissue engineering include Matrigel, collagen type I matrix, a collagenchitosan blend, and collagen sponges, and several of the synthetic biomaterials used for fat tissue engineering include polyglycolide (PGA) scaffolds, poly(ethylene glycol)-based hydrogel and perfluoroelastomer ${ }^{[14]}$. Natural biomaterials are widely preferred because of their biocompatible and biodegradable nature.
\end{abstract}

Currently, chitosan, a marine polysaccharide, is the most widely used biopolymer in various biomedical applications because of its potential in stimulating hemostasis and accelerating the regeneration of damaged or lost tissues in the process of wound healing ${ }^{[15]}$. Naturally obtained chitosan biomaterials are more 
biocompatible and biodegradable than synthetic materials ${ }^{[16-18]}$. Chitosan-based biomaterials have now been of major interest because of their anti-microbial, nontoxic, renewable, bio absorbable and biopolymer properties. Chitosan is a cationic amino polysaccharide which exhibits a stronger adherence to the tissues at the site of wound that are anionic. Various types of chitosan biomaterials in the forms of hydrogel, pastes, powders, sheets, sponges and porous scaffolds are produced using different processing methods ${ }^{[19]}$. Therefore, surface modification of chitosan scaffolds to increase the biofunctionality, is an effective approach in clinical applications. Chitosan can be fabricated into a stable, porous scaffold and hence, numerous populations of cultured cells could be seeded onto these scaffolds. Tissue-engineered porous chitosan scaffolds (PCSs) act as an artificial extracellular matrix (ECM) that lays the foundation for cell attachment, cell proliferation, cell differentiation and the migration of cells to the desired site to repair damaged tissues. Porous chitosan skin-regenerating templates have been found to be a more biocompatible wound dressing due to their stimulation of cell proliferation $^{[18,20]}$. This study highlights the structural morphology and proliferative capacity of ASCs seeded on the customized PCSs.

\section{Materials and methods}

\subsection{Biomaterial}

The PCSs used in this research were customized and produced by the Advanced Materials Research Centre, Standards and Industrial Research Institute of Malaysia (SIRIM). The procedure for preparing chitosan was mentioned previously ${ }^{[21]}$. Briefly, Ultrapure and medical-grade chitosan were produced from prawn shell. Chitosan powder with molecular weight of $634 \mathrm{kDa}$ and a degree of deacetylation of $89 \%$ was purchased from Hunza Nutriceuticals, Malaysia. $2 \%(\mathrm{w} / \mathrm{v})$ of chitosan solution was prepared by dissolving chitosan powder in $1 \%(\mathrm{v} / \mathrm{v})$ reconstituted acetic acid. The $\mathrm{pH}$ of chitosan solution was adjusted to 6.2 by adding $0.2 \mathrm{M}$ sodium bicarbonate. Porous chitosan scaffolds were prepared by freeze-drying technique whereby chitosan solution was poured into graded porous mould and cooled to $-20^{\circ} \mathrm{C}$ before lyophilization for $24 \mathrm{~h}$. They were sterilized by ethylene oxide gas and stored at room temperature, in the dark and dry place. The PCSs were characterized and analyzed by scanning electron microscopy (SEM) and Fourier transform infrared (FTIR) spectroscopy, as described in a previous article ${ }^{[22]}$.

\subsection{Isolation and culture of Adipose-Derived Stem Cells}

Adipose tissue samples were obtained from subcutaneous adipose tissue resected from patients with informed written consent. Ethical clearance was obtained from the Human Ethics Committee of Universiti Sains Malaysia (Reference no.: USMKK/PPP/JEPeM [236.3(16)]). The samples were processed within $24 \mathrm{hrs}$. Each tissue sample was washed several times with Dulbecco's phosphatebuffered saline (DPBS) (Gibco, USA) containing 1\% (v/v) antibioticantimycotic (Gibco, USA) to remove any blood from the resected tissue. The tissue was then chopped finely with sterile scissors and enzymatically dissociated by the addition of $0.1 \%(\mathrm{w} / \mathrm{v})$ collagenase type I (Worthington, USA), followed by overnight incubation at $37^{\circ} \mathrm{C}$. The next day, the enzymatically dissociated tissue was passed through a $70 \mu \mathrm{m}$ cell strainer to remove the remaining undissociated tissue, neutralized by the addition of DPBS and centrifuged at $800 \mathrm{x} \mathrm{g}$ for $5 \mathrm{~min}$. The obtained stromal cell pellet was resuspended in Dulbecco's modified Eagle's medium (DMEM) (Gibco, USA) containing 10\% MSC-qualified fetal bovine serum (FBS) (Gibco, Mexico) and $1 \%$ antibiotic-antimycotic solution. The cells were cultured in T25 flasks and maintained in a 5\% carbon dioxide $\left(\mathrm{CO}_{2}\right)$ incubator at $37^{\circ} \mathrm{C}$. Once the primary cultures reached $80 \%$ confluence, further passages were obtained by detaching the cells from the flasks using TrypLE Express (Gibco, USA). Ten minutes after the addition of TrypLE Express, the detached cells were neutralized by adding DMEM and were centrifuged at $800 \mathrm{x} \mathrm{g}$ for $5 \mathrm{~min}$. The obtained stromal cell pellet was resuspended in Dulbecco's modified Eagle's medium (DMEM) (Gibco, USA) containing 10\% MSC-qualified fetal bovine serum (FBS) (Gibco, Mexico) and $1 \%$ antibiotic-antimycotic solution. The cells were cultured in T25 flasks maintained in a 5\% carbon dioxide $\left(\mathrm{CO}_{2}\right)$ incubator at $37^{\circ} \mathrm{C}$. Once the primary cultures reached $80 \%$ confluence, further passages were obtained by detaching the cells from the flasks using TrypLE Express (Gibco, USA). Ten minutes after the addition of TrypLE Express, the detached cells were neutralized by adding DMEM and were centrifuged at $800 \mathrm{x}$ g for 5 min. The obtained cell pellet was then resuspended in DMEM and cultured in new T25 flasks. The ASCs were maintained in MesenPRO RS Medium (Gibco, USA), which is specifically formulated for the growth of MSCs used from passage 3 onward. ASC cultures at passages 3-6 were used throughout the experiments.

\subsection{Characterization of Adipose-Derived Stem Cells}

\subsubsection{Immunocytochemistry (ICC) analysis}

The experiment was performed using an IHC Select ${ }^{\circledR}$ HRP/DAB Test (Chemicon, USA). Cells were cultured on a 24-well plate at a seeding density of $1 \times 10^{6} / \mathrm{ml}$. Within the next two days, the ASCs reached $80 \%$ confluence, and the staining was performed. The cells were washed with DPBS twice and were fixed with cold methanol for $15 \mathrm{~min}$ at $4^{\circ} \mathrm{C}$. The non-specific binding sites on the cells were blocked with goat serum for $10 \mathrm{~min}$. Primary antibodies, with an optimized dilution ratio of 1:500, were added to the cells and incubated for $1 \mathrm{hr}$ at room temperature (RT). The primary antibodies that were used to stain the ASCs were mouse anti-human immunoglobulin G1 (IgG1) monoclonal antibodies against CD29 (Clone 12G10, Abcam, UK), CD105 (Clone SN6, Abcam UK), CD73 (Clone AD2, BD Pharmingen, NJ) and CD90 (Clone 5E10, BD Pharmingen, NJ). Mouse IgG1 (1:500) (Abcam, UK) was used as the negative control. The cells were washed using washing buffer and were incubated with secondary antibody (biotinylated goat antimouse IgG) for $10 \mathrm{~min}$. Streptavidin-horseradish peroxidase (HRP) was then added to bind to the biotin-labeled secondary antibody for $10 \mathrm{~min}$ of further incubation. After the addition of the streptavidinHRP, the cells were washed with washing buffer and stained with chromogenic reagent to label cells that were positive for the abovementioned markers for $10 \mathrm{~min}$. Finally, the cells were counterstained with hematoxylin solution for $1 \mathrm{~min}$, washed with washing buffer and analyzed under an inverted phase-contrast microscope (Zeiss, Germany). All of the reagents except for the primary antibodies were provided in the kit (the IHC Select ${ }^{\circ}$ HRP/DAB Test).

\subsubsection{Flow cytometry analysis}

Surface marker staining was performed for flow cytometry analysis. Cells were detached from their flask using TrypLE Express, resuspended at a concentration of $1 \times 10^{6} / \mathrm{ml}$ and stained with primary antibodies for $1 \mathrm{hr}$. The primary antibodies used were the same antibodies as those used for the ICC analysis, but with different dilutions: mouse anti-human IgG1 monoclonal antibodies against CD90 (1:100), CD29 (1:50), CD73 (1:50), and CD105 (1:10). Purified mouse IgG1, k (1:1000) (Clone MOPC-21, BD Pharmingen, $\mathrm{NJ}$ ) was used as an isotype control. An antibody against the hematopoietic stem cell marker CD34, or PE mouse anti-human CD34 (1:100) (Clone 563, BD Pharmingen, NJ), was used as a negative control. The cells were then washed with DPBS and centrifuged at $800 \mathrm{x} g$ for $5 \mathrm{~min}$. Next, all cells except the negativecontrol cells were stained with FITC-conjugated goat polyclonal secondary antibody to mouse IgG H\&L (1:1000) (Abcam, UK) for 30-40 $\mathrm{min}$ at RT. The cells were then analyzed using a flow 
cytometer (FACSCanto II, BD Biosciences, USA). The data acquisition and interpretation were performed using BD FACSDiva software.

\subsection{Induced cell differentiation}

\subsubsection{Detection of lipids}

ASCs at passage 4 were cultured at a seeding density of $1 \times 10^{5} /$ well in a six-well plate. The ASCs were maintained in MesenPRO RS Medium until the cells reached $80 \%$ confluence. The StemPro Adipogenesis Differentiation Kit (Gibco, USA) was used to induce the ASCs to differentiate into adipocytes. The cells were maintained in adipogenic differentiation medium for 14 days, during which the medium was changed every 3 days. The control groups were maintained in adipogenic differentiation medium for 3 days, and after that, MesenPRO Medium was used. The differentiation was confirmed using Oil Red O staining. Briefly, the differentiated cells were removed from the incubator after 14 days and were washed with DPBS. After the DPBS was removed, the cells were fixed with $10 \%$ neutral buffered formalin for $1 \mathrm{hr}$ at RT. The formalin was then removed from each well, and the cells were washed with deionized (DI) water. Next, 60\% isopropanol was added to each well and incubated at RT for 10 min to extract the lipids from the adipose cells. Once the isopropanol was removed, and Oil Red O staining solution (Sigma, USA) was added and incubated for $10 \mathrm{~min}$ at RT. The cells were then washed several times with DI water until the Oil Red O stain was completely removed. The cells were counterstained by adding hematoxylin solution for $1 \mathrm{~min}$, after which the hematoxylin was removed and the cells were washed with DI water. Approximately $1 \mathrm{ml}$ of DI water was finally added to cover the entire cell monolayer, and images were then captured using an inverted phase-contrast microscope.

\subsubsection{Osteoblast detection}

ASCs at passage 4 were cultured at a seeding density of $1 \times 10^{5} /$ well in a six-well plate. The ASCs were maintained in MesenPRO RS Medium until the cells reached $80 \%$ confluence. The StemPro Osteogenesis Differentiation Kit (Gibco, USA) was used to induce the ASCs to differentiate into osteoblasts. The cells were maintained in osteogenic differentiation medium for 21 days, during which the medium was changed every 3 days. When the differentiated cells were removed from the incubator after 21 days, the differentiation was confirmed by Alizarin Red S staining and alkaline phosphatase (AP) staining. For the Alizarin Red S staining, the differentiated cells were washed with DPBS, fixed with $10 \%$ neutral buffered formalin for $1 \mathrm{hr}$, stained with Alizarin Red S staining solution (Millipore, USA) in the dark for $45 \mathrm{~min}$ at RT and analyzed using an inverted phase-contrast microscope. For the AP staining, the differentiated cells were washed with DPBS and fixed with $10 \%$ formalin for less than 1 min because a longer incubation would inactivate AP. The cells were then stained with 5-bromo-4-chloro-3-indolyl-phosphate with nitro blue tetrazolium (BCIP/NBT) substrate solution (Sigma-Aldrich, USA) at RT in the dark for 5-10 min and washed with DI water, after which images were observed using an inverted microscope.

\subsubsection{Detection of cartilage extracellular matrix}

The ASCs at passage 4 were cultured at a seeding density of $1 \times 10 \%$ well in a six well plate. The ASCs were maintained in MesenPRO RS Medium until the cells reached $80 \%$ confluence. The StemPro Chondrogenesis Differentiation Kit (Gibco, USA) was used to induce the ASCs to differentiate into cartilage spheroids. The ASCs were maintained in the chondrogenesis differentiation medium for 14 days, during which the medium was changed every 3 days. When the differentiated cells were removed from the incubator after 14 days, they were washed with DPBS. The cells were then fixed with $10 \%$ formalin for $1 \mathrm{hr}$. After that, the cells were washed with DI water and stained with $2 \mathrm{ml}$ of Alcian-Blue staining solution (Millipore, USA) overnight at RT in the dark. On the subsequent day, the staining solution was removed, and the cells were washed with destaining solution (ethanol (98-100\%): acetic acid (98-100\%) at a dilution ratio of $3: 2$ ) for $20 \mathrm{~min}$. The destaining solution was removed, and DPBS was added to cover the cell monolayer, after which images were observed using an inverted phase-contrast microscope.

\subsection{Incorporation of ASCs into PCSs and cell viability assessment}

Chitosan discs that were $2 \mathrm{~mm}$ thick and $5 \mathrm{~mm}$ in diameter were used. The PCSs were placed on 24-well plates, and wells with only ASCs were used as a control. ASCs at passage 4 were seeded on the PCSs at a density of $2 \times 10^{6} / \mathrm{ml}$. The viability assay was performed from days 1-7. The cells were incubated with PrestoBlue cell viability reagent (Invitrogen, USA) at a dilution ratio of $1: 10$ for 24 hrs, and then the supernatant was transferred to a 96-well plate and analyzed using the Infinite 200 PRO NanoQuant enzyme-linked immunosorbent assay (ELISA) reader (Tecan, Austria). The absorbance was determined at $570 \mathrm{~nm}$, with a reference wavelength of $600 \mathrm{~nm}$.

\subsection{Morphological analysis}

\subsubsection{Scanning electron microscopy}

Following the seeding of ASCs on the chitosan scaffolds for 1, 3 or 7 days, the PCSs were washed and fixed with $2.5 \%$ glutaraldehyde for $1 \mathrm{hr}$. The scaffolds were then washed and dehydrated using a graded alcohol series with dilutions of $30 \%, 70 \%$ and $100 \%$ ethanol. SEM (FEI Quanta 450 FEG, Netherlands) was used to observe the morphology of the ASCs attached to the PCSs.

\subsubsection{Hematoxylin and Eosin $(H \& E)$ staining}

The interaction of ASCs with the PCSs were further analyzed using H\&E staining. On day 7, the PCSs seeded with ASCs were washed and fixed with $10 \%$ formalin, embedded in paraffin and sectioned at a $4 \mu \mathrm{m}$ thickness. H\&E staining was then performed as follows. Sections of scaffold seeded with ASCs were carefully transferred to dry slides. The slides were washed in xylene to remove the paraffin. The slides were then sequentially washed in $100 \%, 95 \%, 80 \%$ and $70 \%$ ethanol for $5 \mathrm{~min}$ each to remove the xylene. Next, the slides were rinsed in tap water for $5 \mathrm{~min}$ to remove the ethanol and immersed in hematoxylin for $15 \mathrm{~min}$ to stain the cell nuclei. Following the hematoxylin staining, the slides were washed in $1 \%$ acid alcohol for 5 secs and in running tap water for $5 \mathrm{~min}$ to remove the excess stain. The slides were then dipped in ammonia for 5 secs to neutralize the reactivity of the acid alcohol, followed by washing in running tap water for $5 \mathrm{~min}$ and immersion in eosin for $5 \mathrm{~min}$ to stain the cytoplasm. Finally, the slides were sequentially washed in $100 \%, 95 \%, 80 \%$ and $70 \%$ ethanol for $5 \mathrm{~min}$ each and in xylene for 3 min. Coverslips were then mounted onto the slides. Images were observed using Mirax Desk scanner (Zeiss, Germany).

\subsection{Statistical analysis}

Statistical analysis was performed using SPSS software, version 21. All values are presented as the mean \pm standard error of the mean. Statistical analysis were done using Kruskal-Wallis test with $\mathrm{n}=4$ and $\mathrm{p}<0.05$ was considered to indicate a statistically significant difference. 


\section{Results}

\subsection{ICC characterization of ASCs using selective markers}

Characterization of the human ASCs was performed using ICC. The cells were stained and found to be positive for the cell surface markers CD29, CD90, CD105 and CD73, as shown in Figure 1. ASCs have spindle shape like morphology.

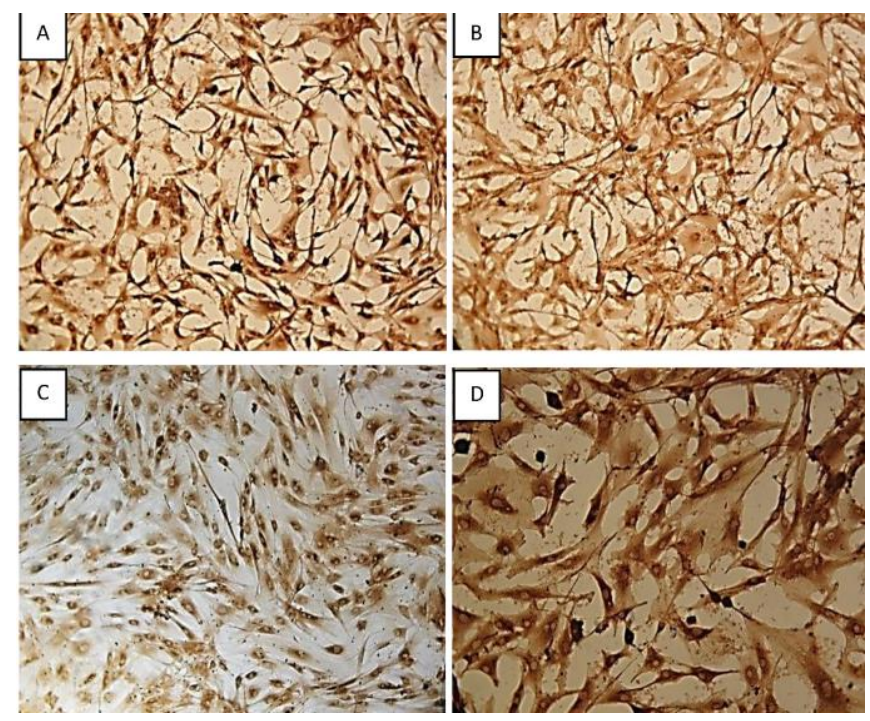

Figure 1. ICC characterisation of ASCs: Isolated ASCs from adipose tissue were positively stained as follows: [A]. CD 29, [B].CD 90, [C]. CD 105 and [D]. CD 73. The morphology of the cells expressing the cell surface markers were captured under $100 x$ magnification.
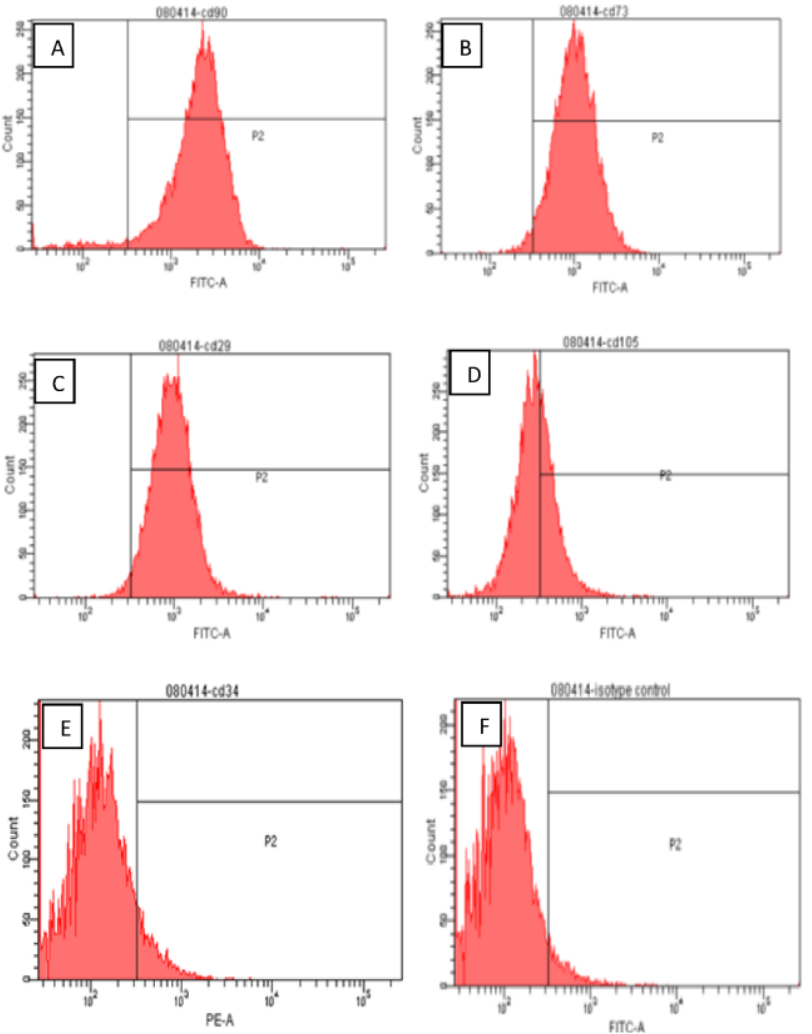

Figure 2. Flow cytometric surface marker expression profile: The side scatter (SSC) and forward scatter (FSC) were adjusted accordingly to gate the population of interest. The gate Pl in the SSC vs. FSC plot, represents the experimental population of ASCs. The gate P2 is referred as the interval gate enclosing the positive cell population in each fluorescence parameter. The positive expression levels of cell surface markers in human ASCs were clearly shown in the figures: [A]. CD 90 (85\%), [B]. CD 73 (89\%), [C]. CD $29(85 \%)$ and Only35\% of ASC population express [D]. CD 105. Also, ASCs were found to be negative for [E]. CD34 (8.8\%). [F]. Isotype control IgG1.

\subsection{Flow cytometry analysis of ASCs}

Flow cytometry analysis of the human ASCs confirmed the cells' expression of the cell surface markers CD90, CD73, CD29, and CD105. The ASCs were found to be negative for the hematopoietic stem marker CD34. These characterized human ASCs were fully capable of differentiating into adipogenic, osteogenic and chondrogenic cell lineages. The samples were analyzed by flow cytometry with at least 10000 events detected, excluding cell debris and aggregates.

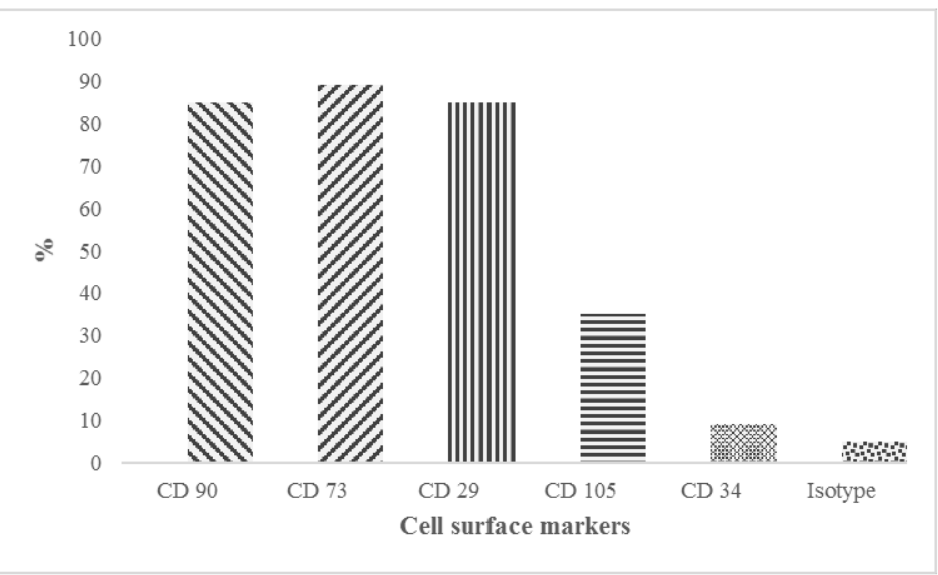

Figure 3. Expression level of cell surface markers: This graph shows the percentage of cells expressing cell surface markers $C D$ 90, CD 73, CD 29, CD 105, CD 34 and Isotype control.

\subsection{Induced differentiation of ASCs into adipocytes, osteoblasts and chondrogenic matrix}

The induced differentiation of the ASCs into adipocytes, chondrocytes and osteoblasts is shown in Figure 4. In figure 4A and $4 \mathrm{~B}$, adipogenic differentiation was observed at 2 weeks after induction, and the differentiation was confirmed using Oil Red O staining. The staining clearly revealed the presence of lipid droplets in the differentiated adipocytes. Alizarin Red S staining and AP staining were performed to confirm calcium deposition and osteoblast formation upon osteogenic induction. While staining the calcium deposits with Alizarin Red S, (Figure 4C) the undifferentiated ASCs without extracellular calcium deposits appeared slightly reddish at 2 weeks of induction, whereas (Figure 4D) the ASCs that fully differentiated into osteoblasts appeared bright orange-red in color at 4 weeks of induction. While staining the osteoblasts with AP, (Figure 4E) the undifferentiated ASCs, showing weak AP activity, appeared faintly bluish in color after 2 weeks of induction, whereas (Figure 4F) the fully differentiated osteoblasts, with high AP activity, appeared dark blue-violet or nearly black in color after 4 weeks of induction. Finally, chondrogenic differentiation of the ASCs was confirmed using Alcian-Blue staining. The cartilage ECM appeared dark-blue (Figure 4G) at 2 weeks and (Figure $4 \mathrm{H}$ ) at 4 weeks after induction.

\subsection{Proliferation rate of ASCs on PCSs}

The data obtained from the quantitative analysis of the PrestoBlue viability assay is shown in the table (Table 1). The proliferation rate of the ASCs on PCSs increased gradually from days 1-7. The results indicate that the ASCs were able to attach and proliferate effectively on PCSs. 

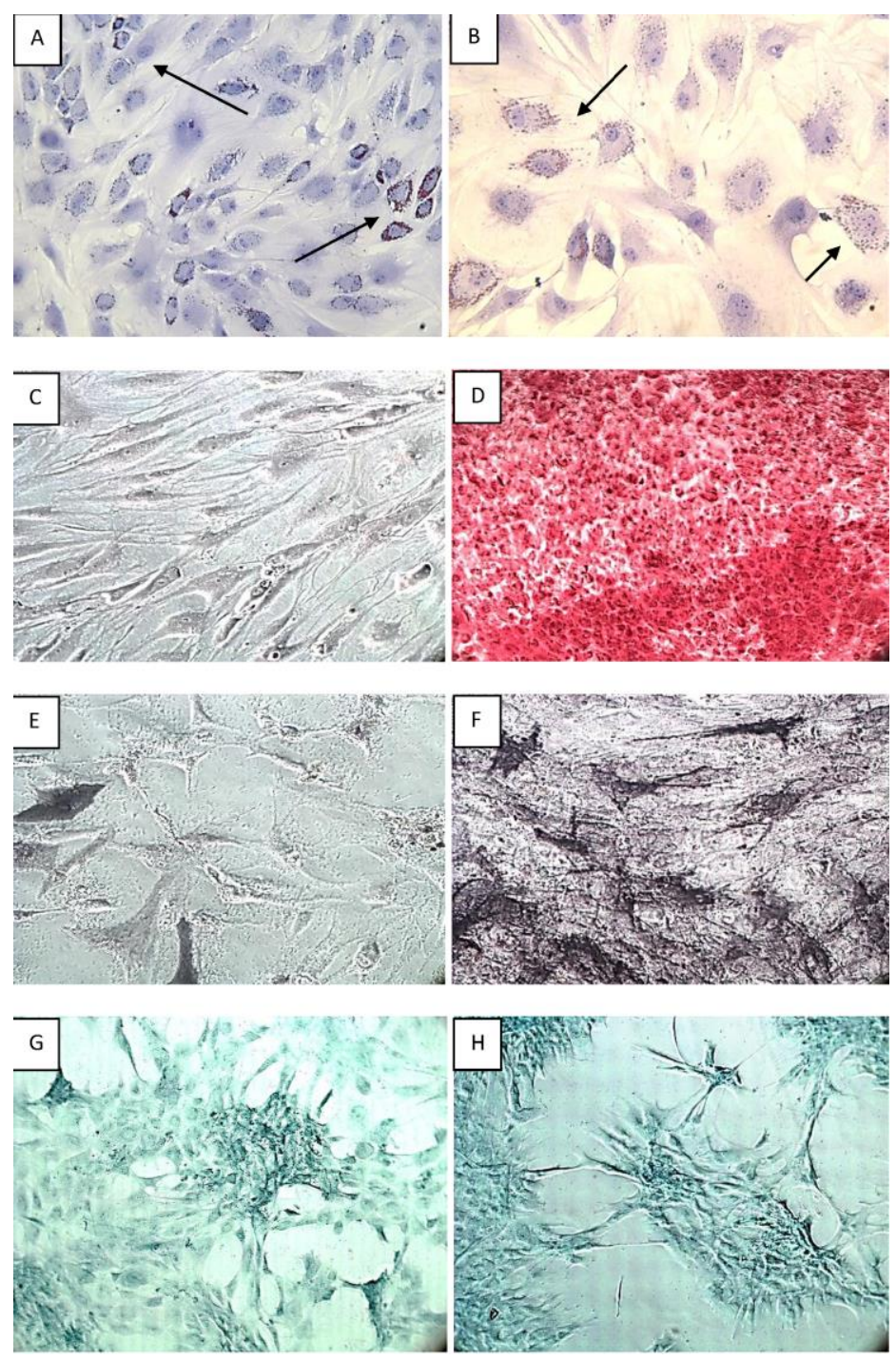

Figure 4. Induced differentiation of ASCs : Adipogenic induction of ASCs at 2 weeks is demonstrated by Oil Red $O$ staining under [A].100x and [B]. 200x magnification. The arrow marks indicate the lipids present in adipocytes which appears red. Osteoblast detection was demonstrated by two types of staining. $i$.) Staining the Calcium deposits with alizarin red S: [C].Undifferentiated ASCs appeared in slightly reddish colour (10x magnification). [D]. Fully differentiated ASCs into osteoblasts appears in bright orange red colour (100x magnification). ii.) AP staining for the detection of osteoblasts showing [E].Undifferentiated ASCs in faintly bluish colour (100x magnification) and [F]. Fully differentiated osteoblasts in black colour (100x magnification). Finally chondrogenic differentiation of ASCs is confirmed using Alcian staining. The cartilage extracellular matrix appears dark-blue [G]. at 2 weeks and [H]. at 4 weeks after induction (100x magnification).

\begin{tabular}{|c|c|c|c|c|c|c|}
\hline DAY 1 & DAY 2 & DAY 3 & DAY 4 & DAY 5 & DAY 6 & DAY 7 \\
\hline $0.53 \pm 0.019$ & $0.74 \pm 0.018$ & $0.96 \pm 0.048$ & $1.15 \pm 0.029$ & $1.30 \pm 0.007$ & $1.41 \pm 0.009$ & $1.43 \pm 0.048$ \\
\hline
\end{tabular}

Table 1. Absorbance at 570nm (mean \pm SEM, $n=4)$ : This figure shows the proliferation rate of ASCs analyzed using PrestoBlue viability assay. The results are presented as mean \pm standard error. The p-value from the Kruskal Wallis test is 0.004 , which is less than 0.05 . Based on the mean ranks from the test, the proliferation rate of ASCs on day 7 is much higher compared to day 1.

\subsection{Observation of cell morphology by SEM}

The SEM analysis is shown in Figure 5. The PCSs were prepared as small discs that were $2 \mathrm{~mm}$ thick and $10 \mathrm{~mm}$ in diameter. (Figure 5A) By SEM, the PCSs seemed to have a round or elongated oval shape with a pore diameter ranging from $39-78 \mu \mathrm{m}$. (Figure $5 \mathrm{~B}$ ) On day 1 , only few cells were found to have adhered to the scaffold, and these cells had a spherical structural morphology. (Figure 5C) On day 3, the cells were found to have an extended cell structural morphology. (Figure 5D) After 7 days of proliferation of the ASCs on the PCSs, the cell numbers had significantly increased, and several of the cells showed fully extended spindle-shape-like morphology.
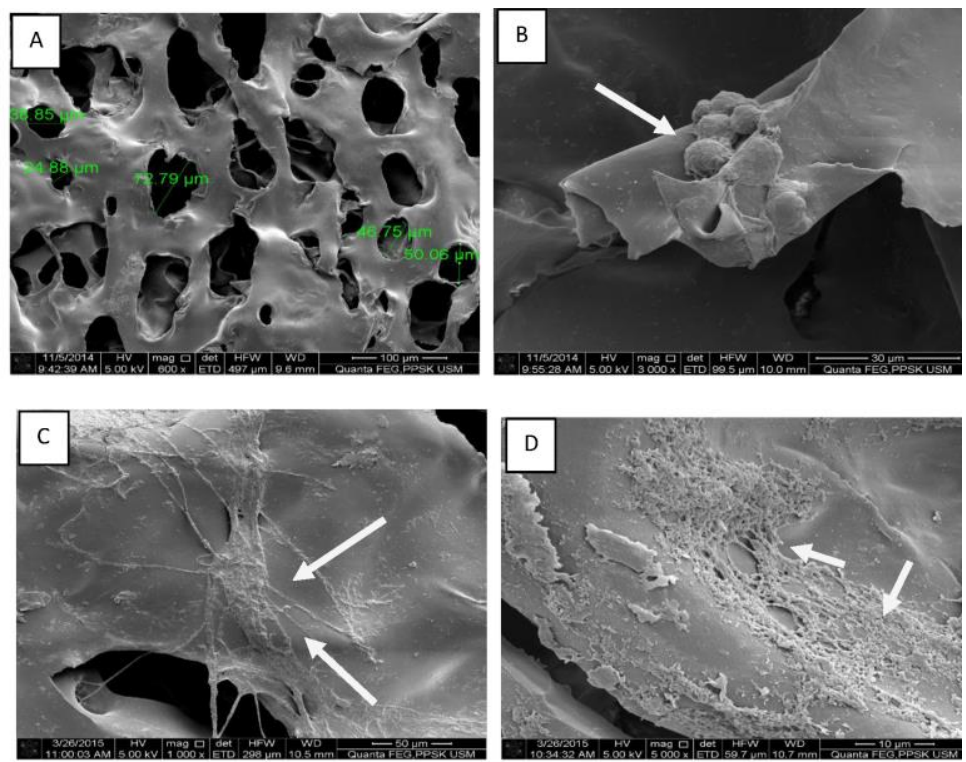

Figure 5. Morphological observation of incorporated ASCs on PCS under SEM: [A]. PCS only without the ASCs (x600). The morphology of ASCs seeded on PCS following: [B]. 24 hours of culture (x3000), [C]. 3 days of culture (x3000) and [D]. 7 days of culture (x12000).

\subsection{Observation of cell morphology by $\mathrm{H} \& \mathrm{E}$ staining}

The ASCs were allowed to proliferate on PCSs for 7 days, and H\&E staining was performed on the $7^{\text {th }}$ day. The nuclei of the cells were stained with hematoxylin, which appeared purple-violet in color, whereas the chitosan scaffold appeared purple-red in color, as depicted in Figure 6. On the 7th day, most of the cells had migrated from the surface of the scaffold to the interior pores of the scaffold, and they were found to be distributed uniformly throughout the PCS.

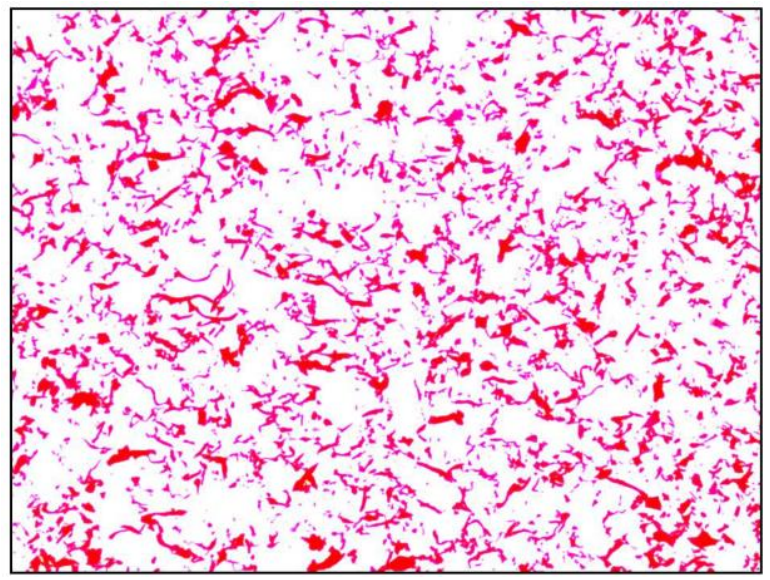

Figure 6. Morphology of ASCs incorporated on PCS observed using $\mathrm{H} \& \mathrm{E}$ staining: $H \& E$ staining on day 7 showed the distribution of ASCs in purple-violet colour and the PCS appears in purple-red colour (100x magnification). 


\section{Discussion}

Wound healing is a complex process that starts with an inflammatory phase, followed by re-epithelialization and remodeling phases ${ }^{[23]}$. In patients with extensive skin loss, complete recovery is often challenging because of a lack of efficient and effective tissue formation during the re-epithelialization phase, leaving disfiguring scars and muscle contractures. Tissue repair requires the migration and proliferation of cells that concertedly communicate with various types of neighboring cells to restore the tissue's function.

As conventional treatment strategies for treating chronic wounds often fails, over the last decade, stem cell-based therapies have been focused on overcoming the difficulties in tissue reconstruction. ASCs derived from subcutaneous adipose tissue are the most promising MSCs; these cells remain stable under normal cell culture conditions, involve less invasive procedures than bone marrow biopsies do and are cost effective. By using specific differentiation media, ASCs can be differentiated into various cell lineages, such as osteoblasts, chondrogenic ECM and adipocytes, to repair damaged cells in the bones or heart or to help to heal chronic ulcers ${ }^{[6.24-26]}$.

The cultured ASCs used in this study were plastic adherent under standard cell culture conditions. These cells' phenotype was positive for the MSC markers CD90, CD73, CD29, and CD105 and negative for the hematopoietic stem cell marker CD34. Identification of the ASC surface immunophenotype is very important to identify and purify the stem cell population. ICC is the most common cell-staining technique and is used to localize the presence of a specific antigen in cells by using a specific primary antibody that binds to the antigen. An added chromogenic dye binds to this primary antibody-secondary antibody-streptavidin complex and converts it into a colored complex, thereby aiding visualization of the cells under a microscope. In the current study, the flow cytometry results revealed that more than $80 \%$ of ASCs expressed the cell surface markers CD90, CD73, and CD29, whereas CD105 was expressed by only $35 \%$ of the stem cell population. Although $\mathrm{CD} 105$ is an important marker for the characterization of MSCs, its expression varies depending on the passage of cells used ${ }^{[27-29]}$.

Studies have shown that ASCs with low or negative expression of CD105 have a greater capacity to differentiate into adipogenic and osteogenic lineages ${ }^{[30,31]}$. The present study clearly showed the expression levels of various markers using fluorescence-activated cell sorting (FACS) analysis and revealed ASCs' capability to differentiate into adipogenic, osteogenic and chondrogenic lineages. The Oil Red O staining revealed lipid droplets in the cytoplasm of the ASCs and confirmed adipogenic induction. The AP staining and Alizarin Red S staining confirmed the osteogenic induction. The Alizarin Red S staining revealed the presence of irregular calcium salt deposits, stained red, and the AP staining revealed black particulate material. The chondrogenic induction was confirmed by Alcian-Blue staining and showed the presence of cartilage ECM, with a dark blue color. The adipogenic, osteogenic and chondrogenic differentiation of ASCs has several clinical applications. Autologous fat tissue graft transplantation is one of the best methods used for tissue reconstruction and plastic surgery. Tissue-engineered fat derived from adipose tissue is used for various reconstructive and cosmetic procedures, including augmentation of the breasts, lips and chin; rejuvenation of aging skin to treat wrinkles; repair of congenital deformities; and also treatment of chronic injuries resulting in soft tissue $\operatorname{loss}^{[32-34]}$. Studies have shown that the osteogenic differentiation of ASCs successfully contributed to bone formation in murine models ${ }^{[35]}$ and that chondrogenic differentiation can be used to generate cartilage for treating degenerative joints in osteoarthritis patients ${ }^{[26]}$. Although there are numerous advantages of ASCs, these cells still need an appropriate scaffold to act as an artificial ECM for cell attachment and proliferation.

There are a number of biomaterials available, but selecting an appropriate scaffold for maximizing the proliferative and differentiative potential of ASCs is very crucial. In one study, it was demonstrated that ASCs derived from Sprague-Dawley rats exhibited greater adhesion and viability when seeded on silk fibroin-chitosan scaffolds ${ }^{[36]}$. Chitosan derived from the exoskeleton of arthropods has been most widely investigated in tissue engineering. There are various grades and derivatives of chitosan used for different clinical applications. Characterization of the different grades of chitosan was conducted in our previous study, and their structural properties were discussed $^{[22]}$. Chitosan-agglomerated scaffolds were found to be excellent scaffolds for the induction of ASC differentiation and proliferation into osteogenic and chondrogenic cell lineages ${ }^{[37]}$. Studies have shown that when human hair follicle stem cells along with fibroblasts were seeded on chitosan scaffolds, these constructs were successful in accelerating the healing of full-thickness wounds in irradiated rats ${ }^{[38]}$. Although numerous studies have been carried out using different types of chitosan, the role of PCSs in the proliferation of ASCs has not been explored. This is the first study to ever evaluate the compatibility of PCSs with the attachment and proliferation of ASCs. ASCs with their greater proliferation capacity and PCSs as an excellent scaffold would play an important role in tissue engineering.

The degree of deacetylation of chitosan used in the fabrication of PCS is greater than $70 \%$ and have a molecular weight of $6.34 \times 10^{5}$ Daltons, that are capable of providing better surface- forming properties $^{[19]}$. Studies have shown that the interconnected pores of a scaffold play an important role in tissue regeneration and also help to achieve greater, more uniform cell attachment and proliferation on the scaffold ${ }^{[39-41]}$. Thus, PCSs, with their porous structure, enable the accumulation and proliferation of a greater number of ASCs. In the current study, the ASCs exhibited greater adhesion starting from 24 hrs after the ASCs were seeded on PCSs. On day 3, changes in the structure of the ASCs were noted, and on day 7, there was an increase in the cell number, and the ASCs had appropriate cellular morphology. The majority of the ASCs were found to have proliferated on the interior of the porous scaffold membrane. The H\&E staining on the $7^{\text {th }}$ day also showed a uniform distribution for a large number of ASCs across the PCSs. Supporting these results, the live/dead assay carried out on ASCs seeded on PCS scaffold, also revealed that the ASCs exhibited a great adhesion and viability on PCS in our previous study ${ }^{[42]}$. A statistically significant difference was observed in the PrestoBlue viability assay, indicating that the ASCs exhibited greater adhesion and proliferation starting from day 1 , and these parameters were found to have increased on subsequent days, clearly demonstrating that higher proliferation of ASCs on PCSs was observed on day 7.

In this study, ASCs were successfully isolated and characterized, and their multi-differentiation potential was also clearly demonstrated. PCSs, with their porous structure, were found to act as an excellent template for the ASCs to attach and proliferate. The viability test revealed that the ASCs seeded on the PCSs had an increased proliferation rate indicating that the PCSs are more compatible for the attachment and proliferation of ASCs. This study may lay the foundation for the further investigation of PCSs for tissue reconstruction in the field of skin tissue engineering. 


\section{References}

1. Da Silva Meirelles L, Chagastelles PC, Nardi NB. Mesenchymal stem cells reside in virtually all post-natal organs and tissues. J Cell Sci. 2006;119(Pt 11):2204-13.

2. Markarian CF, Frey GZ, Silveira MD, Chem EM, Milani AR, Ely PB, , Horn AP, Nardi NB, Camassola M. Isolation of adiposederived stem cells: A comparison among different methods. Biotechnol Lett. 2014;36(4):693-702.

3. Ong WK, Tan CS, Chan KL, Goesantoso GG, Chan XHD, Chan E, Yin J, Yeo CR, Khoo CM, So JBY, Shabbir A, Toh SA, Han W, Sugii S. Identification of specific cell-surface markers of adiposederived stem cells from subcutaneous and visceral fat depots. Stem Cell Reports. 2014;2(2):171-9.

4. JK F, Zhu M, Wulur I, Alfonso Z. Adipose-Derived Stem cells. In: Bunnell B, Prockop D, Phinney D, editors. Mesenchymal stem cells: methods and protocols. Humana Press; 2008. p. 59-67.

5. Lee RH, Kim B, Choi I, Kim H, Choi HS, Suh K, Bae YC, Jung JS. Characterization and expression analysis of mesenchymal stem cells from human bone marrow and adipose tissue. Cell Physiol Biochem. 2004;14(4-6):311-24.

6. Mizuno H, Tobita M, Orbay H, Uysal AC, Lu F. Adipose-Derived Stem Cells as a Novel Tool for Future Regenerative Medicine. In: Hayat MA, editor. Stem Cells and Cancer Stem Cells. Springer Netherlands; 2014. p. 165-74.

7. Johnson PJ, Parker SR, Sakiyama-Elbert SE. Controlled release of neurotrophin-3 from fibrin-based tissue engineering scaffolds enhances neural fiber sprouting following subacute spinal cord injury. Biotechnol Bioeng. 2009;104(6):1207-14.

8. Silva NA, Salgado AJ, Sousa RA, Oliveira JT, Pedro AJ, LeiteAlmeida H, Cerqueira R, Almeida A, Mastronardi F, Mano JF. Development and characterization of a Novel Hybrid Tissue Engineering-based scaffold for spinal cord injury repair. Tissue Eng Part A. 2009;16(1):45-54.

9. De Coppi P, Bartsch G, Siddiqui MM, Xu T, Santos CC, Perin L, Mostoslavsky G, Serre AC, Snyder EY, Yoo JJ. Isolation of amniotic stem cell lines with potential for therapy. Nat Biotechnol. 2007;25(1):100-6.

10. Gomathysankar S, Halim AS, Yaacob NS. Proliferation of Keratinocytes Induced by Adipose-Derived Stem Cells on a Chitosan Scaffold and Its Role in Wound Healing, a Review. Arch Plast Surg. 2014;41(5):452-7.

11. Parenteau N, Hardin-Young J, Ross R. Skin. In: Lanza R, Langer $\mathrm{R}$, Vacanti J, editors. Principles of tissue engineering. 2nd ed. Academic Press; 2011. p. 879-87.

12. Nambu M, Kishimoto S, Nakamura S, Mizuno H, Yanagibayashi S, Yamamoto N, Azuma R, Nakamura S, Kiyosawa T, Ishihara M. Accelerated wound healing in healing-impaired $\mathrm{db} / \mathrm{db}$ mice by autologous adipose tissue-derived stromal cells combined with atelocollagen matrix. Ann Plast Surg. 2009;62(3):317-21.

13. Halim AS, Khoo TL, Mohd Yussof SJ. Biologic and synthetic skin substitutes: An overview. Indian J Plast Surg. 2010;43(3):23-8.

14. Niemelä S, Miettinen S, Sarkanen JR, Ashammakhi N. Adipose tissue and adipocyte differentiation: molecular and cellular aspects and tissue engineering applications. Top Tissue Eng. 2008;4(1):26.

15. Hoekstra A, Struszczyk H, Kivekäs O. Percutaneous microcrystalline chitosan application for sealing arterial puncture sites. Biomaterials. 1998;19(16): 1467-71.

16. Keong LC, Halim AS. In vitro models in biocompatibility assessment for biomedical-grade chitosan derivatives in wound management. Int J Mol Sci. 2009;10(3):1300-13.

17. Lim CK, Yaacob NS, Ismail Z, Halim AS. In vitro biocompatibility of chitosan porous skin regenerating templates (PSRTs) using primary human skin keratinocytes. Toxicol Vitr. 2010;24(3):721-7.

18. Lim CK, Halim AS. Biomedical-grade chitosan in wound management and its biocompatibility in vitro. Magdy Elnashar Publ InTech. 2010

19. Periayah MH, Halim AS, Saad AZM. Chitosan: A Promising Marine Polysaccharide for Biomedical Research. Pharmacogn Rev. 2016; 10(19): 39-42.

20. Luangbudnark W, Viyoch J, Laupattarakasem W, Surakunprapha P, Laupattarakasem P. Properties and biocompatibility of chitosan and silk fibroin blend films for application in skin tissue engineering. Sci World J. 2012;2012.
21. Zainol I, Halim AS, Ghani SM, Deraman MA, Saliman MR, Mohamed MSW. Preparation and characterisation of bilayer chitosan membrane as skin regenerating template. Malaysian J Microsc. 2008;14:24-9.

22. Periayah MH, Halim AS, Gomathysankar S, Sukari AAA, Saad AZM, Rashid AHA, Ujang Z, Muslim NZM. N, OCarboxymethylchitosan (NO-CMC) and Oligo-Chitosan (OC): Scaffold Characterization. Int J Basic Appl Sci. 2014;3(4):532-40.

23. Kirsner RS, Eaglstein WH. The wound healing process. Dermatol Clin. 1993;11(4):629-40.

24. Fromm-Dornieden C, Koenen P. Adipose-derived stem cells in wound healing: recent results in vitro and in vivo. OA Mol Cell Biol. 2013;1(8):1-6.

25. Baer PC, Geiger H. Adipose-derived mesenchymal stromal/stem cells: tissue localization, characterization, and heterogeneity. Stem Cells Int. 2012;2012.

26. Locke M, Feisst V, Dunbar P. Concise Review: Human AdiposeDerived Stem Cells: Separating Promise from Clinical Need. Stem Cells. 2011;29(3):404-11.

27. Yamamoto M, Nakata H, Hao J, Chou J, Kasugai S, Kuroda S. Osteogenic Potential of Mouse Adipose-Derived Stem Cells Sorted for CD90 and CD105 In Vitro. Stem Cells Int. 2014;2014.

28. Dominici M, Le Blanc K, Mueller I, Slaper-Cortenbach I, Marini FC, Krause DS, Deans RJ, Keating A, Prockop DJ, Horwitz EM. Minimal criteria for defining multipotent mesenchymal stromal cells. The International Society for Cellular Therapy position statement. Cytotherapy. 2006;8(4):315-7.

29. Barry FP, Boynton RE, Haynesworth S, Murphy JM, Zaia J. The monoclonal antibody $\mathrm{SH}-2$, raised against human mesenchymal stem cells, recognizes an epitope on endoglin (CD105). Biochem Biophys Res Commun. 1999;265(1):134-9.

30. Choy L, Skillington J, Derynck R. Roles of autocrine TGFBreceptor and Smad signaling in adipocyte differentiation. J Cell Biol. 2000;149(3):667-82.

31. Levi B, Wan DC, Glotzbach JP, Hyun J, Januszyk M, Montoro D, Sorkin M, James AW, Nelson ER, Li S, Quarto N. CD105 protein depletion enhances human adipose-derived stromal cell osteogenesis through reduction of transforming growth factor $\beta 1$ (TGF- $\beta 1$ ) signaling. J Biol Chem. 2011;286(45):39497-509.

32. Khouri R, Del Vecchio D. Breast reconstruction and augmentation using pre-expansion and autologous fat transplantation. Clin Plast Surg. 2009;36(2):269-80.

33. Katz AJ, Llull R, Hedrick MH, Futrell JW. Emerging approaches to the tissue engineering of fat. Clin Plast Surg. 1999;26(4):587603.

34. Patrick CW. Tissue engineering strategies for adipose tissue repair. Anat Rec. 2001;263(4):361-6.

35. Cowan CM, Shi Y-Y, Aalami OO, Chou Y-F, Mari C, Thomas R, Quarto N, Contag CH, Wu B, Longaker MT. Adipose-derived adult stromal cells heal critical-size mouse calvarial defects. Nat Biotechnol. 2004;22(5):560-7.

36. Ji W, Zhang Y, Hu S, Zhang Y. Biocompatibility study of a silk fibroin-chitosan scaffold with adipose tissue-derived stem cells in vitro. Exp Ther Med. 2013;6(2):513-8.

37. Malafaya PB, Pedro AJ, Peterbauer A, Gabriel C, Redl H, Reis RL. Chitosan particles agglomerated scaffolds for cartilage and osteochondral tissue engineering approaches with adipose tissue derived stem cells. J Mater Sci Mater Med. 2005;16(12):1077-85.

38. Mohd Hilmi AB, Halim AS, Jaafar H, Asiah AB, Hassan A. Chitosan dermal substitute and chitosan skin substitute contribute to accelerated full-thickness wound healing in irradiated rats. Biomed Res Int. 2013;2013.

39. Ma PX. Biomimetic materials for tissue engineering. Adv Drug Deliv Rev. 2008;60(2):184-98.

40. Ma PX, Choi J-W. Biodegradable polymer scaffolds with welldefined interconnected spherical pore network. Tissue Eng. 2001;7(1):23-33.

41. Zeltinger J, Sherwood JK, Graham DA, Müeller R, Griffith LG. Effect of pore size and void fraction on cellular adhesion, proliferation, and matrix deposition. Tissue Eng. 2001;7(5):55772.

42. Mohamed M, Gomathysankar S, Mat Saad A, Noorsal K, Halim A. Viability of Adipose-derived Stem Cells (ASCs) on Porous Chitosan Scaffold. Int J Pharm Sci Res. 2015;1(9):3781-7. 


\author{
Abbreviations \\ MSCs: Mesenchymal Stem Cells \\ ASCs: Adipose-Derived Stem Cells \\ WAT: White Adipose Tissue \\ ECM: Extracellular Matrix \\ PCS: $\quad$ Porous Chitosan Scaffold
}

\title{
Potential Conflicts of Interests
}

\section{None}

\section{Acknowledgments}

The work related to this paper was financially supported by a Research University Grant (1001/PPSP/813058) from Universiti Sains Malaysia. We thank Advanced Materials Research Centre, Standards and Industrial Research Institute of Malaysia (SIRIM) for providing PCSs. We would like to thank Mr. Baharudin Zakaria and Mr. Mohd Anizan Bakhtiar Abdullah from the School of Health Sciences (USM), who assisted in performing the SEM analysis. We would also like to thank Dr. Muhammad Hassan from the School of Medical Sciences (USM) for assisting in the H\&E staining.

\section{Corresponding Author}

Ahmad Sukari Halim, Reconstructive Sciences Unit, School of Medical Sciences, Universiti Sains Malaysia, 16150, Kubang Kerian, Kelantan, Malaysia; E-mail: ashalim@usm.my 